\title{
COMPLEX STRUCTURES IN ROLE AND REFERENCE GRAMMAR: SOME PROBLEMATIC CASES
}

\author{
Ana Ortigosa Pastor \\ Universidad de La Rioja
}

\begin{abstract}
This paper addresses the issue of complex structures in the framework of Role and Reference Grammar (Van Valin \& LaPolla, 1997). These authors establish an explicit and clear-cut typology of complex constructions for English highlighting the role of operators (modality, aspect, tense...etc.). However, after analyzing a 1000-example corpus, several cases will turn out to be quite ambiguous and not so definite as regards clausal selection. Consequently, such examples will prove that the boundaries between complex structures as established by Van Valin and LaPolla (1997) cannot be considered as so limited and precise, and that they will usually have need of a more detailed account drawing evidence from other theoretical grounds.
\end{abstract}

\section{INTRODUCTION}

Within the framework of Role and Reference Grammar (Van Valin and LaPolla 1997), complex sentence constructions are explained according to the units which are involved and the relationship among those units. As is the case with simple sentences, the nucleus (predicate), core (predicate + arguments) and clause (predicate + arguments + non-arguments) constitute the main building blocks (Van Valin \& LaPolla 1997: 441) in complex constructions. Among the relationships between these three units, coordination and subordination represent the two traditional nexus in the field of linguistics; in a co-ordinate construction, as its name implies, the constituents of each clause are independent from each other, sometimes joined by a coordinating 
conjunction. ${ }^{1}$ In a subordinate construction, on the other hand, one of the clauses, usually introduced by a subordinating conjunction, is dependent on a main one and at the same time, functions as a modifier or argument of this main clause. Apart from these two traditional relations, Van Valin and LaPolla (1997), elaborating on Olson (1981), add a third one called cosubordination ${ }^{2}$ which will be revised below.

Adhering to Van Valin and LaPolla's terminology, three levels of juncture "units" (nuclear, core and clausal) and three nexus types -relations(coordination, subordination and cosubordination) are distinguished. Each of these sets in turn, will be combined with the other yielding seven juncturenexus constructions in English ${ }^{3}$ : nuclear cosubordination, core coordination, core cosubordination, core subordination, clausal coordination, clausal cosubordination and clausal subordination. In order to explain and justify the previous examples, the role of operators such as tense, aspect, modality...etc. in complex sentences will be of paramount importance. ${ }^{4}$

In this paper, after having analyzed the results displayed by a 1000example corpus drawn from the British National Corpus, I show that there are some cases of these complex structures which are quite ambiguous and present several doubts as regards clausal selection. The discussion will proceed as follows: first, I critically revise the complex structures typology as established by Van Valin and LaPolla (1997), emphasizing the role of operators in such constructions; likewise, these structures are illustrated by several examples from the corpus. Then, I comment on the results of a 1000-example corpus research as regards complex structures where some doubtful cases concerning clausal selection are explained in further detail.

1. Van Valin and LaPolla (1997: 454) stress the need to differentiate coordination, an abstract syntactic relation between units in a juncture, from conjunction, a specific construction type of the general form ' $\mathrm{X}$ conj $\mathrm{Y}$ ', which can be an instantiation of coordinate or cosubordinate nexus.

2. Van der Auwera (1997: 3) points out to two relevant features for distinguishing nexus relations: [+ dependent] and [+ embedded]. In the case of cosubordination, this author states that a clause entity is cosubordinate if it cannot occur by itself [+dependent] and it is not part of another clause [-embedded].

3. All three nexus types are possible at all three levels of juncture, therefore there are nine possible juncture-nexus types in universal grammar. However, a language does not need to have all nine, and while English displays seven juncture-nexus types for instance, Korean appears to have all nine.

4. The concept of operators was originally introduced in the Functional Grammar framework by Dik (1978) and further developed by Hengeveld (1989). In this way operators are defined as the grammatical means through which more information about the state of affairs can be added. Each operator, in turn, must affect a specific layer of the clause. For further information I refer the reader to Dik (1997a) and Van Valin \& LaPolla (1997). 


\section{THE ROLE OF OPERATORS IN COMPLEX STRUCTURES}

As stated above, operators play a significant role in the understanding of complex constructions. In this sense, operator dependence, i.e. obligatory sharing of operators across the units in a juncture, constitutes the distinguishing feature of cosubordination. In Van Valin and LaPolla's words (1997: 455), "the non-matrix unit(s) must be dependent upon the matrix unit for the expression of at least one operator at the level of juncture". In the remainder of this section the focus will be on the role of operators in the seven juncture-nexus constructions found in English. ${ }^{5}$

Thus, beginning with nuclear cosubordination, a nuclear juncture consists of a single core made up of two different nuclei, which functions as a single complex predicate taking a single set of core arguments: e.g. Mary left the door open. Likewise, and since we are talking about cosubordination, both nuclei must also share the relevant operators of a nuclear juncture, namely (nuclear) directionals, (nuclear) negation and aspect. Examples of nuclear cosubordination structures from the corpus are illustrated by (1):

(1)

a. AL2 $409^{6}$. Despite its cost-cutting and job reduction programs, British Petroleum went out of its way yesterday to keep shareholders happy at the group's annual meeting.

b. HWN 609. Don't forget to put your bin out today.

In (1a) the nuclear cosubordination relation holds between the verbal predicate to keep and the adjectival predicate happy, which share the two core arguments British petroleum and shareholders. In (1b), we find again two nuclei: the verbal predicate to put and the prepositional predicate out; both predicates share the argument you (though in this case is omitted since it is an imperative clause) and the argument bin.

With respect to core juncture structures, a general feature of this kind of junctures is that one of the arguments must function as the semantic argument for the two nuclei in each core. In order to distinguish a core juncture from a nuclear juncture two more characteristics should be mentioned: the first one is that a core juncture may require a complementizer, in this case to, whereas nuclear junctures do not permit one; and the other is that, while the two nuclei

5. For this section, I have thoroughly revised Van Valin and LaPolla's (1997: 455) account on complex structures and many of their examples are provided.

6. Whenever I use examples from the BNC corpus the reference 'code', constitued by numbers and letters, will be specified. 
can appear adjacent in a nuclear juncture (e.g. Take off your shoes), this is not possible in a core juncture (e.g. He persuaded Amy to leave _ ${ }^{*}$ He persuaded to leave Amy).

In a core cosubordination construction like He tried to wash the car there are two different cores, in which the two nuclei, tried and wash share one argument $\mathrm{He}$, and, as it is a cosubordination nexus, both cores must also share the relevant operators for a core juncture: modality, (core) directionals and internal negation. Examples from the corpus are given in (2):

(2)

a. K1X 3483. Police want to talk to anyone who saw the lorry, the taxi or a white Cavalier Sri in the area earlier today.

b. B08 2049. You may want to invite former colleagues to work for or with you in your new sphere.

In (2a) the core cosubordination structure holds between the predicates want and talk, which share the argument police. In (2b) the predicates want and invite are also in a cosubordinate relation sharing the argument you; in this case, we find the realization of the core operator for modality, expressed by may, which must be shared by both cores.

Operator dependence constitutes a crucial feature for distinguishing core cosubordination from core coordination. Let us consider the following examples:

(3)

a. He tried to wash the car.

a'. He didn't try to wash the car.

b. Pat told Mary to wash the car.

b'. Pat didn't tell Mary to wash the car.

In (3a'), an instance of core cosubordination, the negation must affect both cores: He didn't try that and, consequently He didn't wash the car.

In (3b), an instance of core coordination, there are again two different cores, in which both predicates, told and wash, share one semantic argument (Mary). However, in this case the core operators are not shared by both cores; hence, in (3b') the negation affects just the first core Pat didn't tell Mary, but this does not imply that Mary did not wash the car finally. The syntactic representation for (3a') and (3b) is given in the following figure: 

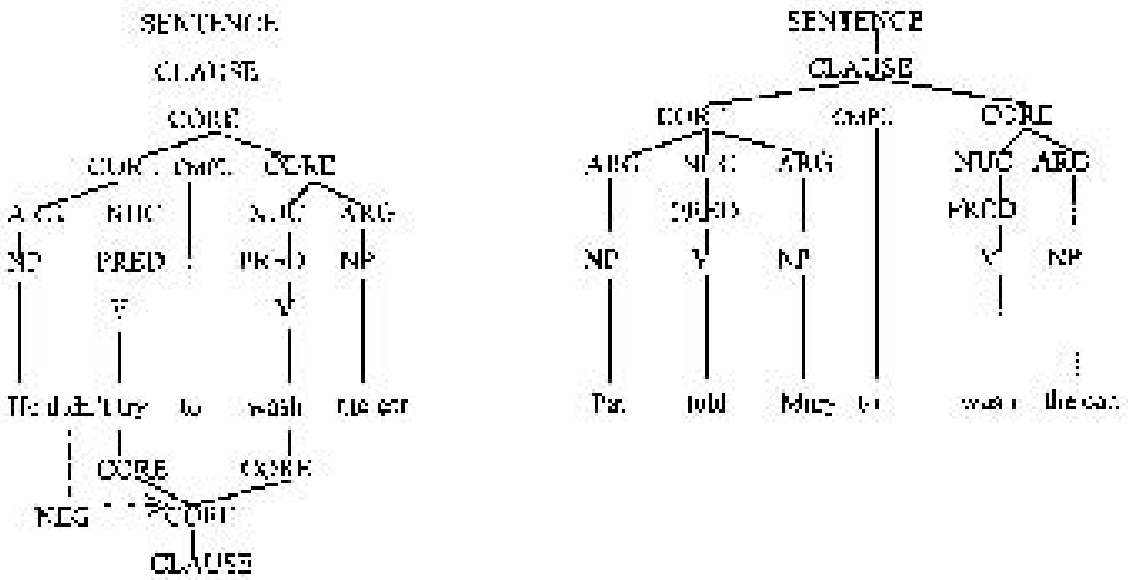

Figure 1. Core cosubordination and core coordination in English.

As regards core subordination constructions, this relationship consists of a core juncture, where there is no sharing of an argument between the two nuclei, but instead, one of the cores functions as an argument of the matrix core:

(4)

a. Maria regretted Peter's losing the game.

a'. Peter's losing the game was regretted by Maria.

b. That Tom won the race surprised Mary.

b'. It was Tom's winning the race that surprised Mary.

Both (4a) and (4b) represent instances of core subordination constructions. In (4a) the subordinate core Peter's losing the game functions as an argument of the matrix core regretted. In (4b) on the other hand, That Tom won the race constitutes a clause which in turn is a core argument (subject) of the main predicate surprised. Functioning as a core argument of a matrix core implies that they may be cleft (4b'), and passivized (4a').

Focusing our attention now on clausal junctures, a clausal cosubordination construction is a sentence made up of two different clauses, both of them sharing any of the clausal operators, namely tense and illocutionary force (IF). Examples from the corpus are given in (5):

\section{(5)}

a. HGF 1994. (...)"I'll see you tomorrow and tell you everything".

b. CEM 2309. Mr Koenig, 44, celebrated with champagne yesterday and rehired five workers he had been forced to sack from his tool business. 
In the previous examples tense and illocutionary force must be shared by each clause. Thus, the clauses in (5a) have the same tense, future, and the same IF, declarative. Both clauses in (5b) also share a declarative IF and tense, in this case past.

With respect to clausal subordination, the two main types involved in this kind of construction are adverbial clauses and that-complement clauses. Adverbial clauses are usually part of the periphery and they modify the matrix core. The subordinating conjunction functions as a predicate and takes a clausal argument. This may be exemplified by (6), where the adverbial clauses are highlighted in bold type:

(6)

a. CH6 1290. A man was shot twice yesterday as he fled from five masked youths.

b. E9U 218. If we win the next three matches we could still go through and we will have a much stronger side.

As regards that-complement clauses, they could be considered as subordinate clauses of core subordination structures since, in the same way, they function as a core argument (this time object) of the matrix core. However, it is at least questionable if object that-clauses are part of the core or fall outside of it; evidence for this fact comes from the placement of peripheral adverbs before the that-clause. Peripheral adverbs must follow all core elements in English; however, if we place a peripheral adverb after an object that-clause the resulting sentence sounds quite unnatural and may yield ambiguous interpretations. This is illustrated by the following examples:

(7)

a. He decided yesterday that he would go to the party.

b. ? He decided that he would go to the party yesterday.

If peripheral elements appear between the nucleus and the that-clause, then the that-clause cannot be within the core and must be analyzed as being a direct daughter of the higher clause node. The syntactic representation of subject that-clauses (core subordination) and object that-clauses (clausal subordination) is illustrated by the following figure: 

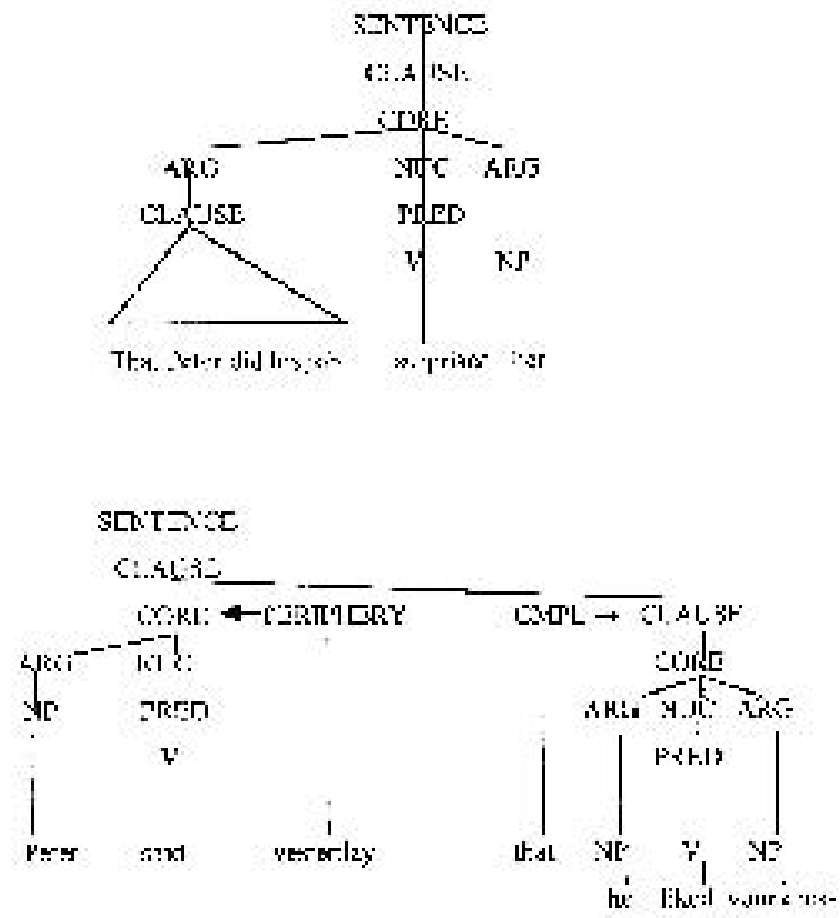

Figure 2. Core and clausal subordination.

The last type of clausal junctures is clausal coordination, a universal juncture-nexus type: e.g. I love 'South Park' but Raquel hates those cartoons; in this case there are two different clauses, both independent from each other, even to the point that each clause may have independent illocutionary force and tense operators.

Nevertheless, in the following section, we will see that there are several cases involving complex structures which are not so precise and definite as the previous ones, and present some doubts as regards clausal selection.

\section{SOME PROBLEMATIC CASES}

After having introduced the main syntactic features of complex constructions, this section will display the results obtained from the analysis of a 1000-example corpus of the occurrence of complex structures selected in a random fashion in groups of 50. This corpus draws from a reliable database, the British National Corpus -hereafter BNC; I consider it a well-suited corpus for my analysis due to its prestige and size. According to Kennedy (1998: 68) 
"studies of many syntactic processes and high frequency vocabulary generally require corpora of between half a million and one million words". The BNC is a corpus of about 100 million words of contemporary spoken/written English from a diversity of sources, hence, and following Kennedy's estimate, it should be enough for the phenomenon under study here.

Most of my analysis will be centered on copula structures, since many of these constructions have resulted to be quite doubtful in terms of the clausal selection guidelines established by Van Valin \& LaPolla (1997).

Before analyzing examples involving copula constructions from the corpus, two cases of non-verbal predicates differentiated by Van Valin and LaPolla (1997) should be considered:

(8)

a. It is likely that Jim will win the race.

b. That Jim will win the race is likely.

Sentence (8b) is a clear example of core subordination where that Jim will win the race functions as a core argument (subject) of the non-verbal predicate likely. However, (8a) is regarded by Van Valin and LaPolla (1997: 482) as an instance of clausal subordination, even though the that-clause is in a subject relation to the predicate likely, and as I said above usually thatsubject clauses are realized by core subordination whereas that-object clauses are realized by clausal subordination structures. One possible explanation for this is the analogy of the that-clause position in (8a) with other types of clausal subordination where the complement clause appears to the right of the sentence, like: He thinks that you should go. Moreover, the subject is doubleinstantiated, it and that he will come, both being co-referential, which makes a clearer division of two different clauses.

A point of reference that should be taken into account when deciding the kind of juncture in non-verbal predication is Hengeveld's classification of copula constructions $(1990)^{7}$. This author classifies copula constructions depending on the type of entities expressed, which can be of four different types:

- First order entity or individual; these entities can have color, size, and weight, can be touched, can be located in space, and can typically be evaluated in terms of their existence.

- Second order entity or state of affairs; these entities can be witnessed and regretted, can be located in space and time, and can typically be evaluated in terms of their reality.

7. For further information see also Hengeveld (1992). 
- Third order entities or propositional content; these entities can be known and forgotten, asserted and denied, can be located in neither space nor time, and can typically be evaluated in terms of their truth.

- Four order entities or speech act; these entities can be uttered and understood, they locate themselves in space and time, and can typically be evaluated in terms of their felicity.

Each of these four entities, in turn, corresponds to a clause layer in Functional Grammar -henceforth FG. Therefore, and elaborating on Lyons (1977), first order entities are expressed by the predicate, second order entities by the predication, third order entities by the proposition and fourth order entities by the clause.

Following the previous classification of entities, Hengeveld (1990: 107) organizes copula constructions according to the type of entity they express. This is illustrated by the following examples:
a. The grass is green.( $1^{\text {st }}$ order)
b. It's stupid to drive without a license. $\left(2^{\text {nd }}\right.$ order $)$
c. It's true that I don't like you.( $3^{\text {rd }}$ order)
d. His question was "Where are you going?" ( $4^{\text {th }}$ order)

Before applying the previous classification to actual examples from the corpus, let us consider first the results of my analyisis as regards copula complex constructions. In this way, these structures can be said to be mainly realized by two juncture-nexus types: 1) core subordination, when a thatsubject clause to the left of the sentence is not co-referential with a pronoun, and 2) clausal subordination, when a that-subject clause to the right of the sentence is co-referential with a previous pronoun. Examples involving copula constructions are given in (10):

(10)

a. FHH 147. That he made a mistake was quite obvious for everybody.

b. BMM 870 It is amazing that we produce any sprinters at all in this country, when you consider the conditions under which we have to race and train.

c. KSH 4620. It was hardly a surprise to the City or anyone else yesterday that Weir Group produced another set of excellent results, with the promise of more to come this year.

According to the distinction drawn above between complex constructions (Van Valin and LaPolla 1997), sentence (10a) corresponds to an instance of core subordination, whereas (10b) and (10c) are examples of clausal subordination. In (10a), that he made a mistake is a core argument of the 
predicate obvious. In (10b) and (10c), that we produce any sprinters at all in this country and that Weir Group produced (both being co-referential with the subject it) constitute the subordinate clauses of the main predicates amazing and surprise, respectively.

As discussed above, Van Valin and LaPolla (1997) also argued that peripheral adverbs, like yesterday, must be placed after all core elements; therefore they justify examples like (10b) or (10c) as cases of clausal subordination due to the position of the adverb after the main clause. Besides, placing the adverb at the end of the sentence would yield ambiguous cases like *He decided that he'll go to Paris yesterday.

Other cases involving copula constructions include examples like:

HHX 11348. It is a terrible indictment of 1991, to think that such events could happen today in Europe.

Sentence (11) is a similar example to those of clausal subordination, with the only exception of to as a complementizer instead of that. If we followed Van Valin and LaPolla complex structures classification, we would be at a loss with an example like the previous one. In this case Hengeveld's classification of copula constructions may be useful. According to this author an expression similar to (11), like It's stupid to drive without a license, expresses a second order entity and this corresponds to the predication layer of the clause. Consequently, it should be considered a case of core juncture since the predication in FG corresponds to the core in RRG, as illustrated by the following table by Van Valin and LaPolla (1997: 47): ${ }^{8}$

\begin{tabular}{lll}
\hline Semantic Unit & RRG layer & FG layer \\
\hline Predicate & Nucleus & Predicate \\
$\begin{array}{l}\text { Predicate }+ \text { Argument } \\
(\mathrm{s})\end{array}$ & Core & Predication \\
$\begin{array}{l}\text { Predicate }+\operatorname{argument}(\mathrm{s}) \\
+(\text { non-arg }(\mathrm{s}))=\operatorname{proposition}\end{array}$ & Clause & Proposition \\
Proposition + DP elements & Sentence & Utterance \\
\hline
\end{tabular}

Table 1. FG and RRG clause layers.

8. When ascertaining the junctures in complex structures by comparing FG/RRG layers, it is only the semantic classification of entities that it is used (that is, operators are not taken into account since some FG operators are distributed differently along the layers). 
However, (11) is not a case of core cosubordination, even though we find the complementizer to, a typical particle of this type of relation according to Van Valin \& LaPolla (1997); this is evidenced by the fact that both predicates terrible indictment and think do not share any core arguments. Hence, it constitutes a case of core subordination, where to think that such events could happen today in Europe functions as a core argument of the predicate terrible indictment. In this way the construction can be cleft as follows:

(11)' It is to think that such events could happen today in Europe that is a terrible indictment of 1991.

Other non-verbal predicate expressions which may present some doubts when deciding the kind of complex structure involved, are headless relative clauses. An example of a headless relative clause taken from Hengeveld (1990: $114)$ is given in (12):

What I mean is that I don't like you.

An expression like What I need is love or I can't remember who Jose saw is a headless relative clause because its structure is quite alike to that of a relative clause like I saw the man who Sam told me about, but with the difference that it does not modify any NP (in the previous sentence the man) since there is not a head noun. According to Van Valin and LaPolla (1997: 503), these headless relative expressions embody clauses that fill a core argument position in the matrix core. But, again, there are similar cases to the one in (11) above like What you need is to go on bolidays, where the kind of nexus involved may present some uncertainties. Following the reasoning for (11), cases like this will be considered instances of core subordination.

Finally, there are several other cases concerning verbal predicate constructions that would require a more exhaustive explanation. These cases are related to clausal subordination structures, namely when these structures express the semantic relation of indirect discourse. Examples of clausal subordination constructions expressing indirect discourse are given in (13):

a. CH6 6604. Yesterday, cuddling three-month-old Jennifer at her home in Bedford, she told how the passionate affair began and how she was left to bring up her daughter on her own.

b. K52 7241. Mrs. Lambird of Belford Gardens, said yesterday (that) she felt (that) the county council should recruit staff trained to deal with dyslexia. 
Example (13a) shows a relation of clausal subordination between the main clause she told and the subordinate clauses how the passionate affair began and how she was left to bring up her daughter on her own. Semantically, as stated above, it is a case of indirect discourse, that is, an expression of reported speech. In this case, the subordinate clauses are introduced by how; these subordinate clauses can be considered as a variation of that-clauses, since in the same way as these, they usually appear to the right of the sentence and they can be passivized, e.g. How the passionate affair began was told by ber.

In (13b) there are two cases of clausal subordination. The first one is again an instance of indirect discourse between the main clause Mrs. Lambird said yesterday and the subordinate clause she felt...with dyslexia. The second subordinate relation expresses a propositional attitude semantic relation and it is realized by the clauses she felt and the county council should recruit staff trained to deal with dyslexia.

However, there are some cases where, even if the main predicates express that semantic relation -indirect discourse-, it is not so clear if the relationship is one of clausal subordination:

\section{(14)}

a. AHF 874. Serbian forces yesterday claimed to have captured the centre of Bijeljina, in norhteastern Bosnia, after days of fierce gun battles with Muslims and Croats.

b. CH3 1982. Graham Taylor yesterday vowed not to quit as England manager but admitted he feels as hounded on the back pages as the Royal Family are on the front.

c.K55 7840. David Holmes, of Ceder Grove, Shildon, was yesterday given an absolute discharge by Darlington magistrates after admitting parking a vehicle in a no waiting zone in Woodland Road, Darlington.

Although the first two cases, (14a) and (14b), represent indirect discourse, they are not examples of clausal subordination. In fact they are two examples of core junctures, since both nuclei in each clause share one argument (in (14a) the nuclei claimed and have captured share the argument Serbian forces and in (14b) the nuclei vowed and quit share the argument Grabam Taylor). With regard to the nexus type, both sentences seem a case of core cosubordination since, apart from sharing a core argument, the complementizer to is also found. Moreover, it cannot be a case of core subordination since none of the cores function as a core argument; neither can it be a coordinate structure since the cores are not independent from each other. If they are core cosubordination constructions, core operators should be shared by the cores in each sentence. However, if an operator of negation is 
added, as in Serbian forces yesterday didn't claim to have captured..., the question arises whether the negation affects both cores, that is, if the fact of not claiming something means not having done it (and the same fact can be applied to vowed); however I do not think this is the case here, since claiming to have achieved something for instance, does not necessarily mean that you have achieved it. Similarly, one may vow to do something but then decide not to do it, or be prevented from doing it. Cases like the previous ones, pose, in my opinion, some doubts as regards the plausibility of cosubordination as a nexus, though I leave this issue as an open question.

Example (14c) is different from the previous ones. In this sentence there is also a verb that usually appears in cases of clausal subordination: He admits that she is not bis girlfriend; however, in this case there is again a core juncture where there is a sharing of the argument David Holmes. Therefore it constitutes another case of core cosubordination, but this time the second core can be cleft and even passivized as the following examples show:

a. It was parking a vehicle that he admitted.

b. Parking a vehicle was admitted by him.

Consequently, it should be concluded that it is an example of core subordination where parking a vebicle functions as a core argument of the main predicate admit.

These previous examples have shown once more that many complex structures are not so explicit and unambiguous as the ones proposed by Van Valin and LaPolla (1997).

\section{CONCLUSION}

In this paper I have attempted to provide a more detailed explanation of several complex structures expressions. In this sense, Van Valin \& LaPolla's (1997) approach is focused on seven definite complex constructions which are justified in relation to specific syntactic features. These seven juncture-nexus types have been explained according to the role of operators such as tense, illocutionary force, aspect, etc., and several cases have been illustrated from the BNC. However, as we have seen, there are several examples which are not so definite and need a more exhaustive and precise analysis. Most of these cases involved copula constructions such as It is a terrible indictment of 1991, to think that such events could happen today in Europe where the kind of juncture involved was not so clear as regards Van Valin \& LaPolla's (1997) typology. In some of these instances, the FG layered clause structure along with Hengeveldís typology of entities proved to be quite useful. Nevertheless, 
such examples showed that the boundaries between complex structures as settled by Van Valin and LaPolla (1997) cannot be regarded as so clear-cut and determined, and that in many cases it is necessary to draw evidence from other theoretical grounds.

\section{REFERENCES}

AUWERA, J. van der. 1997. "Cosubordination", in Working Papers in Functional Grammar (63). Amsterdam: University of Amsterdam.

DIK, Simon C. 1997a. The Theory of Functional Grammar, Vol. 1: The Structure of the Clause. Foris: Dordrecht.

DIK, Simon C. 1997b. The Theory of Functional Grammar, Vol. 2. Complex and Derived Constructions. Edited by K. Hengeveld. Berlin \& New York: Mouton de Gruyter.

FOLEY, W. \& VAN VALIN, R. 1984. Functional Syntax and Universal Grammar. Cambridge: Cambridge University Press.

HENGEVELD, K. 1989. "Layers and Operators in Functional Grammar". Journal of Linguistics, 25, 127-157.

HENGEVELD, K. 1990. "Semantic relations in non-verbal predications", in Nuyts et al, eds., 101-122.

HENGEVELD, K. 1992. Non-verbal predication: theory, typology, diachrony. Berlin \& New York: Mouton de Gruyter.

KENNEDY, G. 1998. An Introduction to Corpus Linguistics. London: Longman.

LYONS, J. 1977. Semantics. Cambridge University Press.

NUYTS, J. et al, ed. 1990. Layers and Levels of Representation in Language Theory. Amsterdam and Philadelphia: John Benjamins.

OLSON, M. L. 1981. "Barai clause junctures: toward a functional theory of inter-clausal relations". PhD dissertation, Australian National University.

VAN VALIN, R. 1993. "A synopsis of Role and Reference Grammar", in Van Valin, (ed.), 1-164.

VAN VALIN, R. \& LAPOLLA, R.J. 1997. Syntax. Structure, Meaning and Function. Cambridge: Cambridge University Press. 